\title{
Quality Monitoring of Soft-Copy Displays for Medical Radiography
}

\author{
Gregory G. Reiker, Nilesh Gohel, Edward Muka, and G. James Blaine
}

\begin{abstract}
As presentation of medical radiographic images on soft-copy displays (cathode ray tubes) becomes increasingly prevalent in electronic radiography, methods of quality assurance must be developed to ensure that radiologists can effectively transfer film-based reading skills. Luminance measurements provide the basis for evaluating the state of soft-copy displays. An integrated approach has been implemented at Mallinckrodt Institute of Radiology (MIR, Washington University, St Louis, MO) that facilitates measurement of geographically distributed soft-copy displays with centralized data logging, performance tracking, and calibration. MIR's central radiology image manager exercises the display station that drives the monitor, harvests the measurement data, stores the results, and submits the resulting data for additional processing. The luminance measurements are collected by a small, portable, photometric instrument designed at MIR that includes a serial port that is accessed via local area terminal service supported by the radiology image manager. The design details of the photometric instrument and example luminance characteristics of several soft-copy displays used at MIR are presented in this report.
\end{abstract}

QOFT-COPY displays such as cathode ray $\checkmark$ tubes (CRTs) are increasingly used in medical radiography for digital imaging procedures such as computed tomography, magnetic resonance imaging, and digital projection radiography (screen/film and storage phosphor plates). ${ }^{1}$ As networks of inquiry and display stations are deployed both inside and outside of the radiography department, it is extremely important that the acquired image data are visualized consistently. ${ }^{2}$ As the perceived brightness of the displayed images becomes standardized, radiologists can be assured that acquired reading skills can be transferred.

To achieve image presentation consistency over time in an imaging network environment, a method of quality control for the luminance measurements, look-up table compensation, and possibly electrical adjustment of CRTs must be considered to assure the temporal stability of the soft-copy displays used in electronic radiology. Unless rigorous and systematic procedures are instituted and followed, there is risk that some image detail may be missed. Additionally, it is possible that new data visualization scales or "target" luminance curves may be proposed that permit radiologists and referring physicians to overcome some of the current limitations of soft-copy displays relative to hard-copy displays. $^{3}$

For the quality-control procedure, we proposed measuring the CRT luminance characteristic curve at regular intervals as a function of digital input value for particular brightness and contrast settings and to harvest the data for subsequent analysis and calibration. To perform the physical measurement, we followed the previous work of others ${ }^{4-6}$ wherein the entire CRT display was set to a particular digital input value while the photodetector was placed a set distance on the axis perpendicular from the center of the surface of the CRT so that it only viewed the central portion $(0.3 \%$ area) of the CRT. With this geometry, the luminance of the CRT was found from the illuminance on the photodetector assuming that the CRT can be considered a Lambertian point source. ${ }^{5} \mathrm{Al}-$ though this measurement procedure, which examines a portion of a uniformly filled CRT screen, has been widely used, ${ }^{3,4}$ we plan to consider other methods that may more closely represent viewing various medical radiographs (T. Gindele, personal communication, October 1991).

It was decided that the quality-control method must be inexpensive, easy to implement, and simple to perform. The cost goal was selected so that all CRTs or at least groups of CRTs in the radiology department could be assigned a photometric instrument. A simple quality procedure would more likely be used by the already challenged staff.

From the McDonnell Douglas Corporation, St Louis, MO; the Mallinckrodt Institute of Radiology, Washington University, St Louis, MO; and Kodak Health Sciences Division, Rochester, $N Y$.

Supported in part by the Kodak Health Sciences Division.

Address reprint requests to Electronic Radiology Laboratory, Mallinckrodt Institute of Radiology, $510 \mathrm{~S}$ Kingshighway, St Louis, MO 63110.

Reprinted with permission from Medical Imaging VI: Picture Archiving and Communications Systems, Society of PhotoOptical Instrumentation Engineers, 1992.

0897-1889/92/0503-0003\$03.00/0 
Our instrument consists of a sensor head, a data-acquisition section, and an interface to a host computer, as shown in the block diagram in Fig 1. A silicon photodiode with a photopic (light-adapted eye) filter was used as the sensor head. The photopic filter/silicon detector combination is positioned relative to the CRT screen with a suction cup assembly. The design also includes a current-to-voltage converter, analogto-digital (A/D) converter, serial link, and power source circuitry for the data acquisition portion. An RS-423 serial port was included to allow data collection via local area terminal (LAT) service. Finally, the measurement process is automated by a computer program that sends commands to the CRT's host processor to display a particular digital input value, waits for the display to stabilize, and then acquires the digitized luminance value from the instrument via the LAT service, converts the data to the appropriate units for the luminance curve, and stores the converted data in a centralized database for future quality tracking and plotting. This luminance instrument with the integrated program provides a simple, systematic approach to monitoring the characteristic curve of CRTs used for radiographic images in a network environment.

\section{DETECTOR AND PHOTOMETRY}

Luminance is measured in candelas per square meter, which is lumens per sterdian per square meter; however, it is often measured in footlamberts. With regard to geometry, luminance is the luminous intensity (candelas) of a surface in a given direction per unit of projected area of the surface as viewed from that direction. ${ }^{7}$ The equation for luminance $(\mathrm{L})$ is given as

$$
\mathrm{L}=\frac{\mathrm{d} \phi}{\mathrm{dAd} \omega(\cos \theta)},
$$

where $\phi$ is the luminous flux, $A$ is the source area, $\omega$ is the solid angle from the source, and $\theta$ is the angle to surface normal. ${ }^{8}$

Luminous flux is related to radiant flux by a wavelength factor developed by the Commissioner International de Clairage (CIE), known as the photopic eye-response curve. ${ }^{9}$ The instrument measures luminance or photometric brightness, which is a measure of the flux emitted (or reflected) from a surface in the visible light range (380 to $760 \mathrm{~nm}$ ) as it appears to the human eye. The peak response of the eye is at $550 \mathrm{~nm}$, where the conversion factor between luminous flux (lumens) and radiant flux (watts) is 680 lumens/W.

For the detector, a silicon photodiode sensor calibrated in photometric units (foot-lamberts or candelas per square meter) is quite sufficient and is typically used for such measurements. For the visible light range ( 380 to $760 \mathrm{~nm}$ ), the silicon photodiode has good responsivity and is extremely linear in the comparison of incident radiant power with photocurrent in amperes. ${ }^{9}$ These sensors, especially the PIN type (United Detector Technology, Hawthorne, CA), show long-term stability, reliability, very low noise, and high impedance, as well as high speed. ${ }^{10}$ The absolute luminance range of 0.0 to 200.0 foot-lamberts for characterizing the CRTs can readily be supported by a silicon sensor used in the photovoltaic mode with the appropriate instrumentation.

The following performance parameters were examined in the selection of the appropriate photodetector:

1. The spectral response curves of the photodiodes' plot wavelength (in nanometers) against responsivity. The source for this instrument is the CRT's phosphors, which have a spectral range of usually 350 to 700 $\mathrm{nm}$ and peak emissions between 420 and
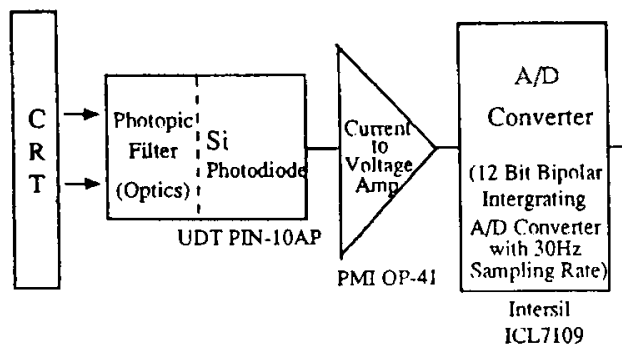

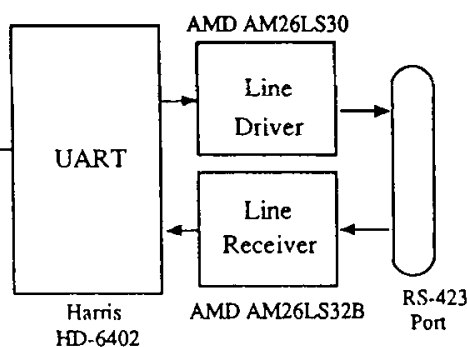

Fig 1. Luminance instrument block diagram. 
$560 \mathrm{~nm}$. However, the response of the eye (photopic) has a spectral range of 380 to $760 \mathrm{~nm}$ with a peak emission at $555 \mathrm{~nm}$.

2. A photodiode's quantum efficiency is a measure of how many photoelectrons are produced for every photon incident on the photosensitive surface. It is defined as $\eta=$ $\left(\mathrm{n}_{\mathrm{e}} / \mathrm{n}_{\mathrm{p}}\right) \times 100 \%$, where $n_{e}$ is the rate of photoelectron generation and $n_{p}$ is the incident photon rate.

3. Photodiode responsivity (or sensitivity) is the magnitude of the electrical signal output from the detector in response to a particular light flux and is given as $\mathrm{S}=\mathrm{i}_{\mathrm{d}} / \phi$ or $\mathrm{S}=\mathrm{V}_{\mathrm{d}} / \phi$, where $i_{d}$ is the detector output current, $V_{d}$ is the output voltage, and $\phi$ is the incident light flux.

4. The minimum detectable power of a photodiode is specified by the parameter noise equivalent power (NEP). The NEP is defined as the radiant flux for which the output signal magnitude equals the noise signal magnitude $(\mathrm{S} / \mathrm{N}=1)$ for a band width of $1 \mathrm{~Hz}$ at a wavelength, usually the peak responsivity. To remove the dependence on band width, NEP is given as an inverse function of the square root of the band width. The noise signal is due to thermal noise generated by the shunt resistance in the photovoltaic mode.

5. The response time parameter of photodiodes is the time taken for the output to respond to a square input pulse of light. The photodiode will be exposed to the light pulses ranging approximately from 350 to 520 microseconds based on its area, and the CRT's line rates and pixel densities.

United Detector Technology's PIN-10AP photodiode with photopic filter was selected for the luminance instrument (Table 1). The PIN$10 \mathrm{AP}$ (with its high zero-bias impedance) is optimized for photovoltaic operation, which has the advantages of low noise and high sensitivity. Therefore, this mode provides better signal-tonoise performance, the best linearity, and good speed (compared with the photoconductive mode), especially at low light levels. The photometric output value can readily be converted to luminance as seen by the human eye. Because the output of the PIN-10AP is proportional to
Table 1. PIN-10AP Properties

\begin{tabular}{lc}
\hline & Parameters \\
\hline Spectral response & $400 \mathrm{~nm}-700 \mathrm{~nm}$ peak at $555 \mathrm{~nm}$ \\
Quantum efficiency & $61 \%$ at $555 \mathrm{~nm}$ \\
Responsivity & $0.272 \mathrm{~A} / \mathrm{W}$ at $555 \mathrm{~nm}$ \\
Noise equivalent power & $1 \times 10^{-11} \mathrm{WHz}-1 / 2$ at $850 \mathrm{~nm}$ \\
Response time & $1.0 \mu \mathrm{s}$ at $0 \mathrm{~V},(\mathrm{C}=10 \mathrm{nf})$ \\
\hline
\end{tabular}

the response of the eye, neither the operator nor the measuring program needs to know the spectral response of the specific phosphor type. This enables the instrument to measure the luminance for any CRT. Values from CRTs using different phosphors may be compared on the same scale of brightness perceived by a human. Table 1 summarizes the important parameters of the detector.

Conversion of the output signal of the PIN10AP to luminance requires knowledge of the geometry between the detector and the source CRT. As stated before, the sensor head was held a set distance from the center of the CRT screen by a suction cup assembly so that this small area could be considered a point source. ${ }^{6}$ When the source is uniformly overfilling the angular aperture of the detector, there is negligible sensitivity to the angle of the detector as long as the full detector angle looks at the source. ${ }^{11}$ Thus, a slight discrepancy in holding the sensor head assembly flush to the curved screen should have a negligible effect. From the mechanical details of the PIN-10AP, the geometry shown in Fig 2 is deduced. The photopic filter is not shown for clarity. Because the PIN-10AP is calibrated for a photopic response in lumens, only the area of the source determined by the front aperture and the solid collection angle (formed by the detector area and the source aperture center) need to be known to convert the value to luminance. Using trigonometry, the full angle of the apex of the

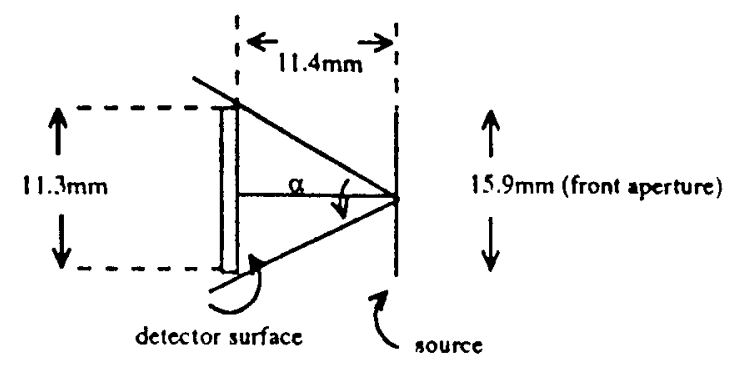

Fig 2. PIN-10AP geometry. 
cone $(\alpha)$ is calculated to be $52.5^{\circ}$, which corresponds to 0.649 steradians for the solid angle. The source aperture area is $1.98 \mathrm{~cm}^{2}$ and $\theta$ is $0^{\circ}$. Starting with the PIN-10AP's nominal responsivity of $0.400 \mathrm{~mA} /$ lumen, the following equation converts diode output to luminance (L) in foot-lamberts:

$$
\mathrm{L}=\mathrm{k} \times \mathrm{I}_{\text {diode }},
$$

where $k=5.69 \times 10^{6}$ foot-lamberts/A and $I_{\text {diode }}=$ photodiode output current.

\section{ELECTRICAL IMPLEMENTATION OF THE INSTRUMENT}

The luminance instrument consists of a sensor head, a data-acquisition section, and an interface to a host computer, as shown in Fig 1. The data-acquisition section was designed using commercially available components for the amplifier, A/D converter, serial link (RS-423), and power source circuits. The components are relatively inexpensive (parts cost $<\$ 500$ in small quantities), so that multiple instruments could be made available to facilitate a program in CRT quality assurance for diagnostic radiology in a network environment. The current instrument was designed for an input range of 0 to 100 foot-lamberts and variable scan rates.

The amplifier circuit uses a JFET operational amplifier (Precision Monolithics Inc, Santa Clara, CA) in the transimpedance mode as a current-to-voltage converter. The amplifier feedback capacitor was selected to prevent gain peaking and instability and, more importantly, to provide a filtering function to integrate the high momentary brightness of the scanning spot as it passes through the area detected by the sensor. Because the source of the peak luminous power is periodic, the relatively long time constant of the feedback filter (approximately 110 milliseconds, corresponding to a $3-\mathrm{dB}$ frequency of $9 \mathrm{~Hz}$, which is same order of magnitude as that of the eye [100 to 200 milliseconds $]^{12,13}$ ) integrates the power received and proportionally indicates the average luminance.

The A/D converter receives the filtered amplifier output voltage, samples it, and converts it to a digital representation. The Intersil ICL7109 (Intersil, Cupertino, CA), which is a highperformance CMOS-integrating A/D converter, was selected. Its output includes 12 data bits, polarity, and over range, which can be accessed in a handshake mode. Integrating the analog input signal over a fixed time interval reduces the noise mean to approximately 0 , especially with this dual-slope configuration. This type of A/D converter has excellent accuracy independent of both the integrating capacitor value and clock frequency because they each affect both the up-slope and the down-ramp in the same proportion. Differential nonlinearity depends only on the counter resolution, and because the output codes are generated by a clock and a counter, there can never be missing codes, because all "counts" inherently exist. ${ }^{14}$ Integrating the input signal smoothes and rejects unwanted high-frequency and $60-\mathrm{Hz}$ noise and averages changes that may occur during the sampling period, including changes due to the scanning spot of the CRT. The A/D converter can perform approximately 7.5 conversions per second. The ICL7109 A/D converter handshakes directly with the serial link block, which consists of a Harris 6402 UART (Harris, Melbourne, FL) and 4702 bit-rate generator. The transfer sequence of 2 data bytes from the instrument takes 2.2 milliseconds including the $\mathrm{A} / \mathrm{D}$ handshake time. Including the read byte requesting the data, the entire cycle would take 3.2 milliseconds, neglecting delays of the host computer, for a rate of 308 readings per second.

\section{INTERFACE TO THE CRT'S HOST COMPUTER}

The quality-control process is automated by a computer program that causes the CRT to display a particular digital input value and waits for the display to stabilize, then acquires the digitized luminance value from the instrument, converts the data to the appropriate units for the luminance curve, and stores the converted data for future quality tracking. The interface method selected is the RS-423 port on the DEC Terminal Server (Digital Equipment Corporation, Hudson, MA), which is connected via Ethernet to a host VAX computer. The DEC Terminal Server connects asynchronous ports to local nodes that implement the LAT protocol. The Terminal Server controls and monitors the communication from the port to the host. A block diagram of the network configuration for the instrument system is given in Fig 3 .

The application program first sets up the 


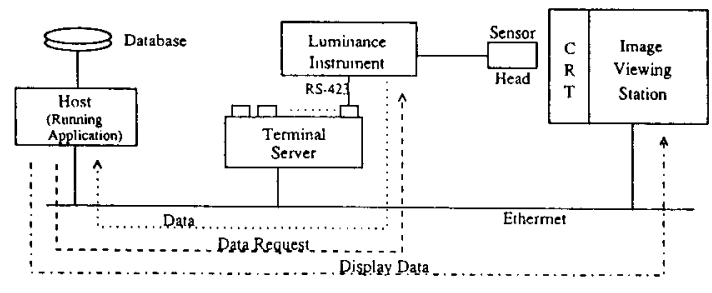

Fig 3. Network configuration for luminance instrument.

LAT port for the instrument before prompting the operator for display information. After the sensor head assembly is mounted a set distance from the CRT screen, the program sends commands (via Ethernet) to initialize the viewing station's screen to digital input value 00 and flushes previous samples from the instrument with multiple data reads. The program reads the luminance measurement data from the instrument at 17 different input values $(00,0 \mathrm{~F}$, $1 F, 2 F, \ldots, F F$ hex). For each of these measurements, the entire screen is filled with a particular digital value (a flat image), and the program delays 2.5 seconds to let the CRT stabilize. Each input value used is displayed four times, and the program reads 10 measurement samples each time an input value is displayed. Therefore, 40 measurement samples are taken and averaged for each input value to improve the estimate of the mean by a factor of 6 . To reduce systematic error, the program randomly selects the order in which each of the 17 different input values will be displayed four times.

The program next converts the averaged raw data to luminance and stores them with the corresponding digital input values in a centralized database. The program then submits the resulting data set to a spreadsheet (Lotus, Cambridge, MA) for management and plotting of the CRT characteristic luminance curve. This CRT characteristic curve (luminance versus digital input value) is for fixed brightness and contrast-control settings. When the program detects a specified deviation from a nominal luminance curve, it logs that the monitored CRT has a quality deficiency. The measurement data will be available from the central facility for future performance tracking.

The entire measurement process takes less than 5 minutes using the method described above. This is based on the $4 \times 17 \times 2.5$-second
CRT delays, the $17 \times 40 \times 3.2$-millisecond data-read cycle delays, and the $17 \times 39 \times$ 133-millisecond read delays. These calculations neglect the delays of the host computer. Consequently, the delay to allow the CRT screen to stabilize is the determining factor in the measurement process time.

\section{INSTRUMENT PERFORMANCE}

The luminance instrument must be stable itself and have a linear relationship with respect to a standard. The United Detector Technology photometer model 350 with a photometric filter and a $15^{\circ}$ lumilens was used as our standard for the luminance measurements. The United Detector Technology model 350 with detector was factory-tested to comply with the published specifications, using laboratory standards that are traceable to the National Bureau of Standards. The model 350 was calibrated to have an accuracy of $1.2 \%$ or 2 counts. For photometric measurements, it is accurate to within $\pm 2 \%$ of the total area under the CIE visibility curve. Its sensor head was also held flush to the screen with a custom holding fixture.

The instrument was tested with the UDT PIN-10AP silicon photodiode and photopic filter on the Imlogix Model 1000 CRT (Imlogix, St Louis, MO). For the luminance measurements of greater than 1 foot-lambert, the instrument's readings agreed with those of the model 350 within $\pm 1 \%$ (percent difference, $(\times 10 \mathrm{AP}-$ $\times 350) / \times 350)$ as shown in Fig 4 . The percent difference varied between $-0.6 \%$ to $+0.9 \%$ for values of greater than 0.5 foot-lamberts. Using linear regression for all the values, the correla-

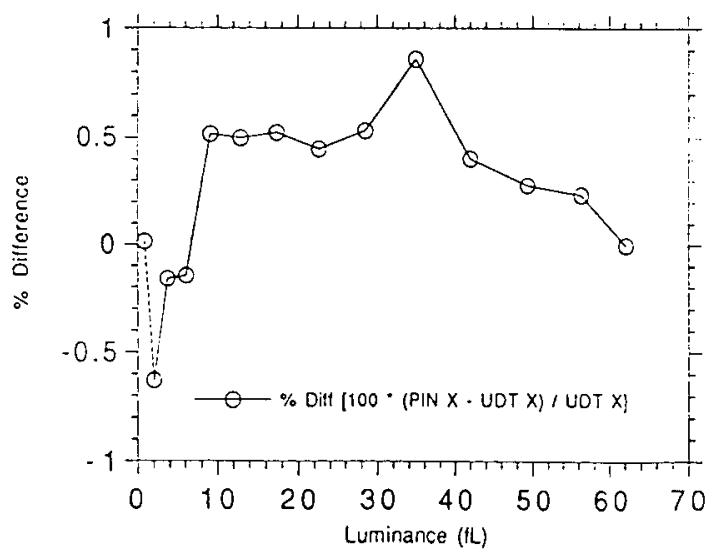

Fig 4. Graph of instrument accuracy. 
tion coefficient is 0.9999921 , which shows quite a linear relationship between the instrument and the model 350 for the range of 0 to 65 foot lamberts. By the method of least squares, the slope of the instrument versus the model 350 is 1.0017 (which is very close to the ideal of 1 ) with a $y$-intercept of 0.0295 (which is very close to the ideal of 0 ).

To test the temporal stability of the luminance instrument, many luminance measurements were taken using both the luminance instrument and our photometer standard over a 2-month period. Results showed the temporal stability to be within the calibration uncertainty described above. The reason for the higher percent differences for luminance values of less than 0.5 foot-lamberts is that systematic errors, such as $A / D$ accuracy, the amplifier offset voltage, bias current, and noise, have greater effects when the filter output voltage is less than $10 \mathrm{mV}$. Therefore, the luminance instrument with the PIN-10AP sensor head showed a very linear relationship in foot-lamberts compared with the standard, which was the model 350.

The noise of the amplifier circuit with the PIN-10AP sensor head and no input light source was measured to be approximately $0.005 \mathrm{mV}_{\mathrm{RMS}}$ at the amplifier output with the Fluke True RMS Multimeter 8062A (Fluke, Everett, WA). Using a $3 \mathrm{~V}_{\mathrm{DC}}$ tungsten-halogen light source to illuminate the PIN-10AP, the noise at the 4.261-V signal level was approximately $3 \mathrm{mV}_{\mathrm{RMS}}$ giving a signal-to-noise ratio (SNR) of 1,420 (note: 12 -bit $\mathrm{A} / \mathrm{D}, 1$ count $=1.2 \mathrm{mV}$ ). At a lower signal level of $0.1101 \mathrm{~V}$, the noise was approximately $1 \mathrm{mV}_{\mathrm{RMS}}$ giving a SNR of 110 . The low noise and high SNR parameters of the instrument showed some of the advantages of using silicon photodiodes in the photovoltaic mode to increase the instrument's sensitivity.

The prototype luminance instrument has been stable throughout the months of testing and showed a very linear relationship with respect to the standard of the model 350 . The instrument was tested in the range of 0 to 100 footlamberts. For measurements of greater than 1 foot-lambert, the instrument readings using the PIN-10AP sensor head agreed with those of the model 350 within $1 \%$. Because the instrument's calculated conversion factor is based on nomi- nal specifications, the application program's conversion factor was adjusted by $1 \%$ to $2 \%$ to calibrate the instrument to the model 350 .

\section{CONCLUSION}

Because CRTs are used more in diagnostic radiology, it is extremely important that the perceived luminance of the displayed images be consistent. To achieve this consistency over time and between CRTs, we have implemented a quality-control procedure that measures the CRT luminance regularly as a function of the digital input value for particular brightness and contrast-control settings. To perform this physical measurement, we have developed a prototype luminance instrument with a sensor head that mounts to the center of the uniform display screen.

This custom-designed luminance instrument offers many advantages over most commercial photometers and radiometers. First, it is relatively inexpensive (sensor head and parts totaling approximately $\$ 500$ ) so that a large number of instruments could be made available to facilitate a program in CRT quality assurance for diagnostic radiology. Also, it is optimized for the range of 0.0 to 100.0 foot-lamberts for CRTs and is accurate within $1 \%$ of a photometer standard after calibration, which is quite sufficient for quality control. In addition to this, it has been stable throughout the weeks of testing and has shown a very linear relationship with respect to the photometer standard. The luminance instrument using the selected sensor with the photopic filter can provide data in footlamberts as perceived by the eye so that data for CRTs of different phosphor types can be compared.

Also, the luminance instrument has a flexible serial interface that allows the quality-control process to be automated. The RS-423 port enables it to be used with the many available serial ports to the host VAX computer. The host computer can then automate the process with a program that causes the CRT to display a particular digital input value and waits for the display to stabilize, then acquires the digitized luminance value from the instrument, converts the data to the appropriate units for the luminance curve, and stores the converted data for 


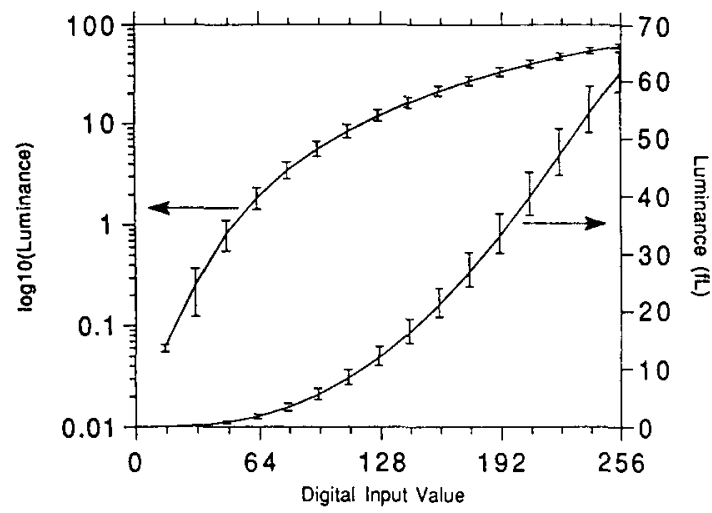

Fig 5. Example test results: average luminance characteristic curves.

future quality tracking and plotting. This implementation offers more centralized control and tracking of the quality data gathered.

As an example, the characteristic luminance curves of seven different Imlogix Model 1000 CRTs versus digital input values were measured by the instrument. The mean values are plotted on both logarithmic and linear scales in Fig 5. The bars on the graphs represent the maximum and minimum values of the seven different CRTs for a given input value. This set of data shows that the variance between CRTs of the same model are small and could be minimized by look-up table compensation. The instrument can be used to gather luminance data, from which the look-up table would be generated, in order to calibrate the CRTs to conform to a "target" luminance curve that would minimize the differences between display units. ${ }^{3}$

The interface program can be expanded to support other CRT display units for this qualitycontrol application. This instrumentation methodology could be used to calibrate the CRTs to conform with a "standard" luminance curve drawn from look-up table compensation after adjustments for brightness and contrast controls. If a display function standard is established, the luminance instrument (with the photopic filter) could assure the consistency between any two CRTs as they try to match the image quality of the hard-copy radiographs by having its application program adjust a look-up table for the input values. ${ }^{4}$ If the calibration and adjustments are made daily, the instrument could be enhanced to take into account the ambient light conditions that could affect the viewing of the radiograph. In conclusion, the prototype luminance instrument effectively implemented the quality control procedure that monitors the CRT luminance as a function of digital input value to assure the consistency and temporal stability of CRTs used in diagnostic radiology.

\section{ACKNOWLEDGMENT}

The authors thank Dr Ronald Evens, Director, and Dr R. Gilbert Jost, Chief of Diagnostic Radiology, both of the Mallinckrodt Institute of Radiology, for their support and encouragement of the activities in this study.

\section{REFERENCES}

1. Cox JR, Muka E, Blaine GJ, et al: Considerations in Moving Electronic Radiography into Routine Use. IEEE J Selected Areas in Comm, 1992 (in press)

2. Cox JR, Moore SM, Whitman RA, et al: Rapid display of radiographic IMAGES. SPIE 1446:40-51, 1991

3. Davros WJ, Gaskil JW, Mun SK: Quality assurance for image display devices in a hospital-wide IMACS network. SPIE 1091:301-304, 1989

4. Blume H, Roehrig H, Browne M, et al: Comparison of the physical performance of high resolution CRT displays and films recorded by laser image printers and displayed on light-boxes and the need for a display standard. SPIE 1232:11-28, 1990

5. Roehrig H, Ji TL, Browne M, et al: Signal-to-noise ratio and maximum information control images displayed by a CRT. SPIE 1232:115-133, 1990

6. Roehrig H, Dallas WJ, Ji TL, et al: Physical evaluation of CRT's for use in digital radiography. SPIE 1091:262-278, 1989
7. Texas Instruments: Optoelectronics and Image Sensors. Data book, 1990

8. Watson J: Optoelectronics. England, Van Nostrand Reinhold, 1988

9. Seippel RG: Optoelectronics for Technology and Engineering. New Jersey, Prentice Hall, 1989

10. United Detector Technology: PIN Series-Planar Diffused Silicon Photodiodes. Data sheets, 1989

11. Oriel Corporation: Light Sources, Monochromators, Detection Systems. Catalog, 1989

12. Tannas LE: Flat Panel Displays and CRTs. England, Van Nostrand Reinhold, 1985

13. RCA, Electro-Optics Handbook. New Jersey, RCA, 1974

14. Bloom S, Wayne S: High-resolution A/D applications and methods proliferate, Electronics Products Magazine, December 12, 1984 